\title{
Anaplastic ganglioglioma originating from the medulla oblongata: case report
}

\author{
Wei Han^, Wei Guan, Jia Shi \\ Department of Neurosurgery, The Third Affiliated Hospital of Soochow University, Changzhou, China \\ Correspondence to: Wei Guan, Jia Shi. No.185, Juqian Street, Changzhou 213003, China. Email: guanwei1402@163.com; 107929159@qq.com.
}

\begin{abstract}
Ganglioglioma (GG) is a rare kind of intracranial tumor with low potential malignancy (WHO grade I), which could occur in any part of the brain. However, a patient with anaplastic ganglioglioma (WHO grade III) in the medulla oblongata is a more peculiar and rare clinical case. In our report, we presented a case of a 45-year-old man with anaplastic ganglioglioma (WHO grade III) in the medulla oblongata, who was perplexed with dysphagia, choking and left-sided numbness for two and a half years. The head magnetic resonance imaging (MRI) showed a $2.7 \mathrm{~cm} \times 2.6 \mathrm{~cm}$ lesion in the medulla oblongata with slightly low signal on T1 weighted image (T1WI) and heterogeneous high signal without calcifications on T2 weighted image (T2WI). The Physical Examination manifested that the patient showed a chronic appearance, along with the damage of oculomotor nerve, glossopharyngeal and vagus nerves and spinal thalamus lateral tract and rope body. Subsequently, the patient underwent surgical and common radiotherapy treatment, which might delay the tumor recurrence and contribute to a better overall survival. Unexpectedly, tentative chemotherapy was carried out when the tumor showed the recurrence trend and anaplastic feature. Our presentation of this case would emphasize the rarity and significance of the diagnosis, differentiation and treatment of such tumors, demanding more clinical cases for exploration.
\end{abstract}

Keywords: Case report; anaplastic ganglioglioma; medulla oblongata; clinical management

Submitted Jan 20, 2021. Accepted for publication Apr 16, 2021.

doi: $10.21037 /$ tcr-21-124

View this article at: https://dx.doi.org/10.21037/tcr-21-124

\section{Introduction}

Ganglioglioma (GG), containing a mixture of welldifferentiated neoplastic ganglion and glial cells, is a rare kind of intra-axial tumors in the central nervous system (CNS) $(1,2)$. GGs can occur in any part of the CNS, including cerebellum, spinal cord, thalamus, hypothalamus, lateral ventricle, and brainstem, which is more commonly supratentorial and is predominantly within the temporal and frontal lobes $(3,4)$. However, there were still a few clinical cases of rarer GGs located in the medulla oblongata $(5,6)$. Although it is universally categorized as World Health Organization (WHO) grade I and accounts for only approximately $1 \%$ of intracranial tumors (3), rarer GGs with a display of anaplastic features have been reported, which could be considered WHO grade III (7). Here, we present a peculiar case with anaplastic ganglioglioma in the medulla oblongata for improvement of the diagnosis, differentiation and treatment of GGs to obtain better outcomes. We present the following article in accordance with the CARE reporting checklist (available at https:// dx.doi.org/10.21037/tcr-21-124).

\section{Case presentation}

\section{History}

The patient was a 45 -year-old male with persistent hiccup,

^ ORCID: 0000-0003-0033-1486. 

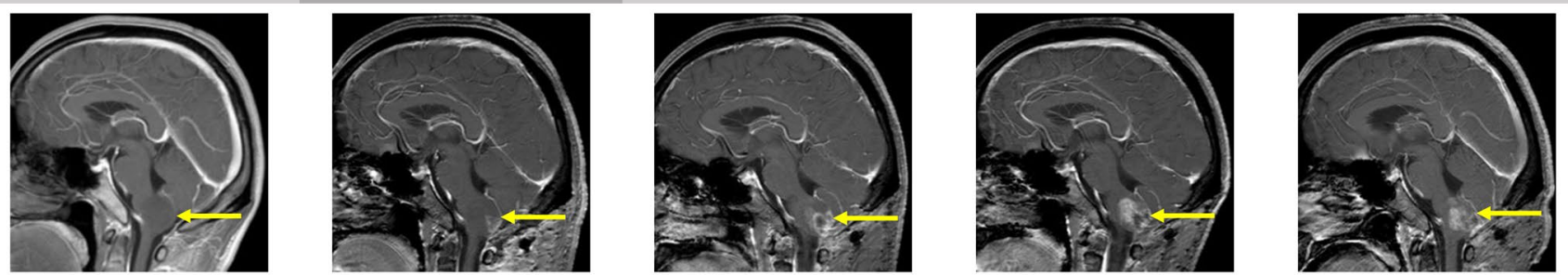

2019/03/12 2019/08/15 2020/02/28 2020/06/18
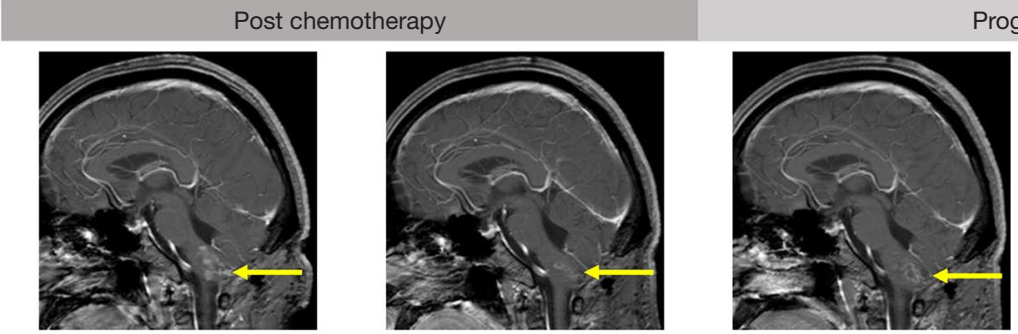

Progression

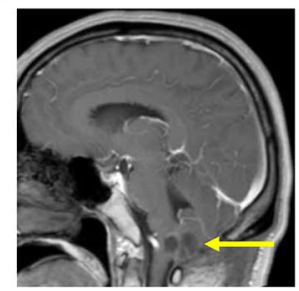

Figure 1 Magnetic resonance imaging (MRI) scans of tumor progression including first diagnosis, post operation, post radiotherapy, post chemotherapy and progression.

hoarseness and repeated vomiting since two years and a half ago. In the following four months, the patient gradually developed dysphagia, choking and left limb numbness, and came to the hospital for further treatment without any other relevant past interventions. Then, the head magnetic resonance imaging (MRI) examination revealed a $2.7 \mathrm{~cm}$ $\times 2.6 \mathrm{~cm}$ occupation of the medulla oblongata (Figure 1). The MRI manifestation showed slightly low signal on T1 weighted image (T1WI) and heterogeneous high signal without calcifications on T2 weighted image (T2WI). On DWI scan, the lesion showed slightly hyperintensity, with an $\mathrm{ADC}$ of $1.98 \times 10^{-3} \mathrm{~mm}^{2} / \mathrm{s}$. The patient was without any medical, family, and psycho-social history including relevant genetic information.

\section{Physical examination}

Generally, the patient showed a chronic appearance. According to the location of the tumor, systematic neurological examination has been carried out. For oculomotor nerve, delayed light reflection was observed in the bilateral pupils. As for glossopharyngeal and vagus nerves, the patient developed hoarse voice, a left uvula sag and decreased left pharyngeal reflex. In addition to these symptoms mentioned above, the damage of spinal thalamus lateral tract and rope body, including superficial sensation in the left limb with muscle strength Grade IV (8) and positive finger-nose and finger-to-finger tests, were also observed.

\section{Operation}

To clarify the pathophysiologic features of the lesion and relieve symptoms, the patient was placed in the prone position and given a partial resection of lesion in the medulla oblongata. An $8 \mathrm{~cm}$ pillow and neck straight incision approach was performed accompanied by a $3 \mathrm{~cm} \times 3 \mathrm{~cm}$ bone window and foramen magnum opening. After the tonsil of cerebellum was gently pulled apart, the medulla oblongata exposed. The lesion in the left of medulla oblongata showed a grey surface and pushed two branches of Posteior Inferior Cerebellar Artery (PICA) to the left. After cutting the cortex of the lesion, there were dense distributions of sickle vessels under the cortex. Considering the protection of the medullary blood vessels and surrounding important tissues, a $1 \mathrm{~cm} \times 0.5 \mathrm{~cm} \times 0.5 \mathrm{~cm}$ mass was removed for pathological examination (Figure 2).

\section{Pathological findings}

The H\&E staining markedly showed that diffuse tumor growth under low magnification and cytonuclear pleomorphism in moderate-density, inhomogeneous cellularity under slightly higher magnification (Figure 3A). The immunohistochemistry suggested: $\mathrm{CgA}(+)$, NeuN 


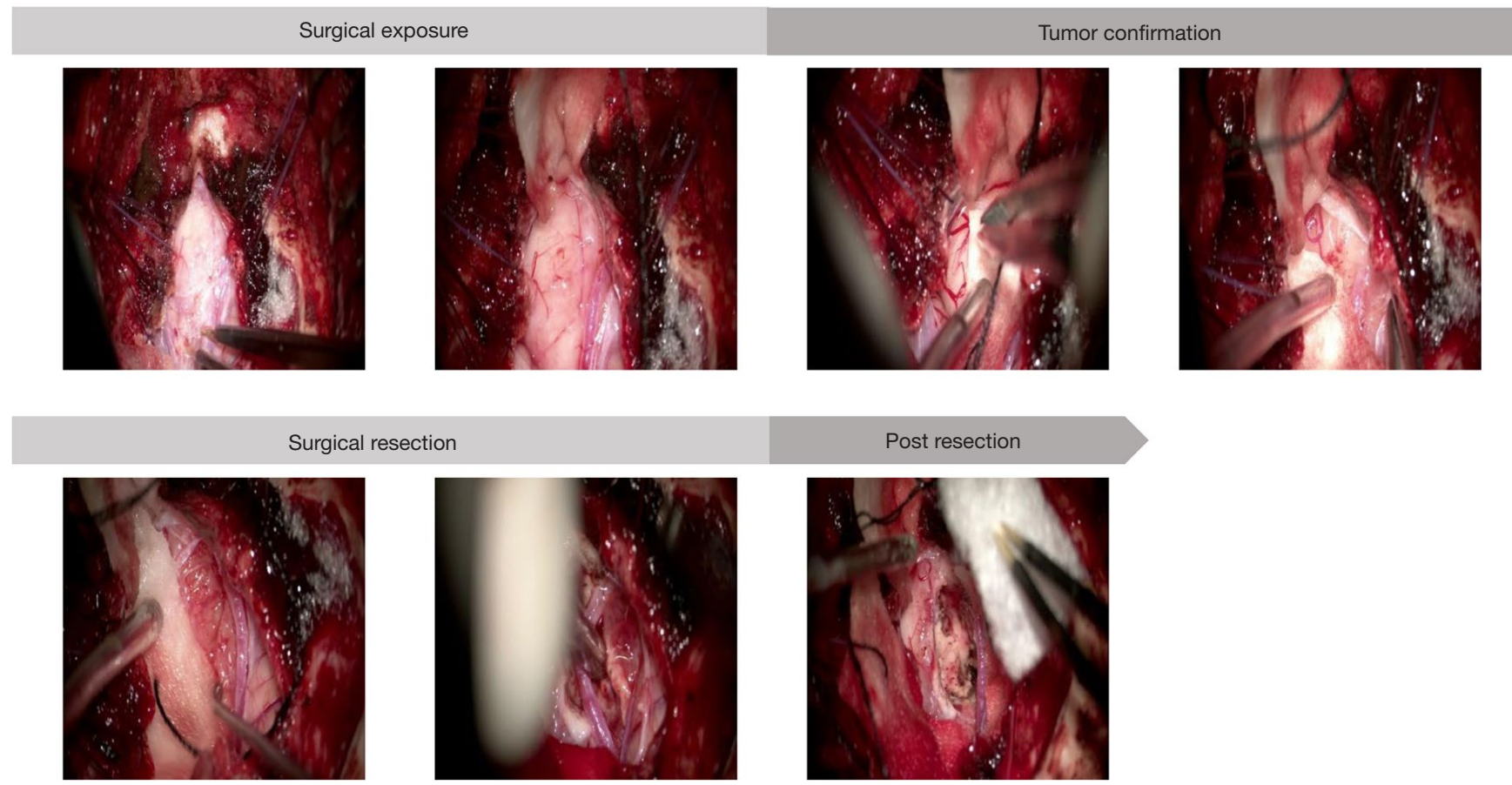

Figure 2 Clinical pictures showing the surgical procedures including surgical exposure, tumor confirmation, surgical resection and post resection.

(+), GFAP (+), S100 (+), IDH1 (-), Nestin (+), Olig2 (+), CD34 (blood vessel+), EMA (-), SOX10 (-), P53 (-), Ki67 (small stove about $5 \%+$ ) (Figure 3B). Given the vital role of BRAF V600E mutation in the migration, invasion and clinical outcomes of GG patients (9), the detecting probe and $\mathrm{qPCR}$ were utilized and the result was negative.

\section{Postoperative outcome}

The patient's postoperative course was uneventful except for transient hiccups and left upper extremity numbness, which vanished in 5 days. 12 days after surgery, the patient with a left upper limb muscle strength Grade V- was discharged from hospital. One month after surgery, the MRI showed a partial resection of tumor in the medulla oblongata with a slightly longer T1 and T2 signal, and a high signal in the FLAIR. No significant enhancement was seen in the enhanced images. The head MRS revealed that Cho, Cr, NAA, Cho/Cr and Cho/NAA were all decreased (Figure 1). The patient was followed by volumetric modulated arc therapy (VMAT) radiotherapy for 22 times in one month. The medullary intracranial mass was $95 \%$ PTV50 $=4,961 \mathrm{cGy} / 25 \mathrm{fx}$, and the medullary neck mass was $95 \%$ PTV45 =4,405 cGy/25 fx (Figure 4).
Three months after surgery, the patient developed nausea, vomiting and headache for two weeks with the head MRI showing a patchy abnormal signal of lesion in the medulla oblongata with a slightly longer $\mathrm{T} 1$ and $\mathrm{T} 2$ signal (Figure 1), and a high signal in the FLAIR. Moreover, the enhanced scan revealed a $2.5 \mathrm{~cm} \times 1.7 \mathrm{~cm}$ ring-enhanced signal. The head MRS showed that Cho, Cr, Cho/Cr and Cho/NAA increased while NAA decreased. In view of the symptoms and imaging examination considering the tumor progression, adjuvant surgery or chemotherapy was recommended due to lacking clinical effective evidence for secondary radiotherapy. While under the patient's insistence, conservative treatment without further surgery or chemotherapy was carried out and some of the symptoms improved.

Six months after surgery, without any progress in related symptoms, compared to the last head MRI, the head MRI had a basically similar imaging performance in T1, T2 phase and FLAIR while the enhanced scan revealed a $2.8 \mathrm{~cm}$ $\times 2.4 \mathrm{~cm} \times 2.3 \mathrm{~cm}$ ring-enhanced signal considering further enhancement of tumor (Figure 1). The head MRS showed that $\mathrm{Cho}, \mathrm{Cr}, \mathrm{Cho} / \mathrm{Cr}$ and Cho/NAA were increased along with Lipase (Lip) peak in necrotic zone while NAA decreased. For further treatment, considering efficacy and 

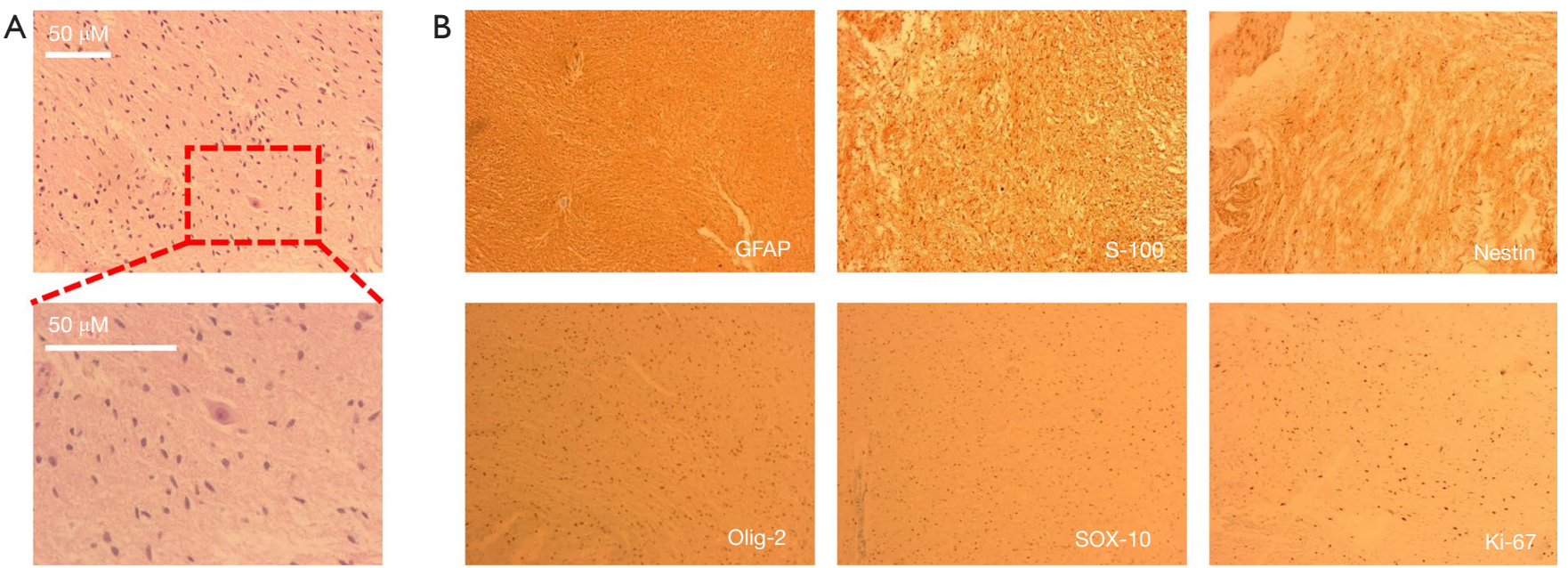

Figure 3 Pathological and immunohistochemical findings of the tumor. (A) H\&E staining (100x) of the lesion are composed of irregular clusters of dysplastic neurons. H\&E staining $(400 x)$ of the lesion shows that the tumorous glial components and the reticulated fiber network are arranged around the astrocytes. (B) Immunohistochemistry (100x) of the lesion showing GFAP (+), S100 (+), Nestin (+), Olig2 (+), SOX10 (-) and Ki67 (small stove about 5\%+).

operation difficulty of immunotherapy, target and electric field therapy, the patient received four courses of oral TMZ $\left(150 \mathrm{mg} / \mathrm{m}^{2} / \mathrm{d}\right.$ for 7 days every 14 days).

Three months after chemotherapy, the head MRI showed that the enhanced scan revealed a $2.4 \mathrm{~cm} \times 2.0 \mathrm{~cm}$ ring-enhanced signal (Figure 1), slightly smaller than before. Eight months after chemotherapy, the imaging performance in T1, T2 phase and FLAIR of the head MRI was similar as the last one, however the ring strengthening further reduces $(1.6 \mathrm{~cm} \times 0.8 \mathrm{~cm})$ (Figure 1), indicating that the tumor was further reduced.

Fourteen months after chemotherapy, however, the head MRI revealed a $1.6 \mathrm{~cm} \times 1.8 \mathrm{~cm} \times 2.0 \mathrm{~cm}$ patch-enhanced signal (Figure 1), with a slightly longer T1 and T2 signal, and a high signal in the FLAIR. Eighteen months after chemotherapy, the head MRI revealed $2.2 \mathrm{~cm} \times 2.3 \mathrm{~cm} \times$ $2.4 \mathrm{~cm}$ patch-enhanced signal (Figure 1), slightly bigger than before. The head MRS revealed that Cho and Cr peaks increased and NAA peak decreased.

At a 33-month follow-up, the patient has slightly left upper limb weakness regardless of hiccup, hoarseness, vomiting, dysphagia and choking. Examination revealed no decreased left pharyngeal reflex, negative fingernose test and finger-to-finger test and only a left upper limb muscle strength Grade V- with a KPS score of 90. Considering the pathological result, the ultimate diagnosis was ganglioglioma in the left medulla oblongata (WHO grade III).
All procedures performed in studies involving human participants were in accordance with the ethical standards of the institutional and/or national research committee(s) and with the Helsinki Declaration (as revised in 2013). Written informed consent was obtained from the patient.

\section{Discussion}

GG is a rare kind of glial-neuronal neoplasm in the CNS with a prolonged survival compared with other intracranial tumors (10). In general, the average age at diagnosis for intracranial GG is from 16 to 29 years (11). Thus, the patient's presentation at 45 in our report is a quite rare occurrence. Moreover, it is acknowledged that GGs could be seen throughout the neuraxis but are most commonly located in the temporal lobes (12). Only 3\% of GGs occur in the medulla oblongata and only one case of pure GGs located in medulla oblongata have been reported in the literature, which were categorized as WHO grade I $(3,6)$. In our study, we present the second case of GG located in medulla oblongata ever reported in the literature, with a WHO grade of III.

Clinically, the main onset symptoms of GGs are epilepsy, increased intracranial pressure with headache, vomiting, and papillary edema (13). Other reported features included limb weakness, gait instability, blepharospasm, diabetes insipidus, and panhypopituitarism (14). Furthermore, it was also reported that GGs often occurred with cerebrospinal 

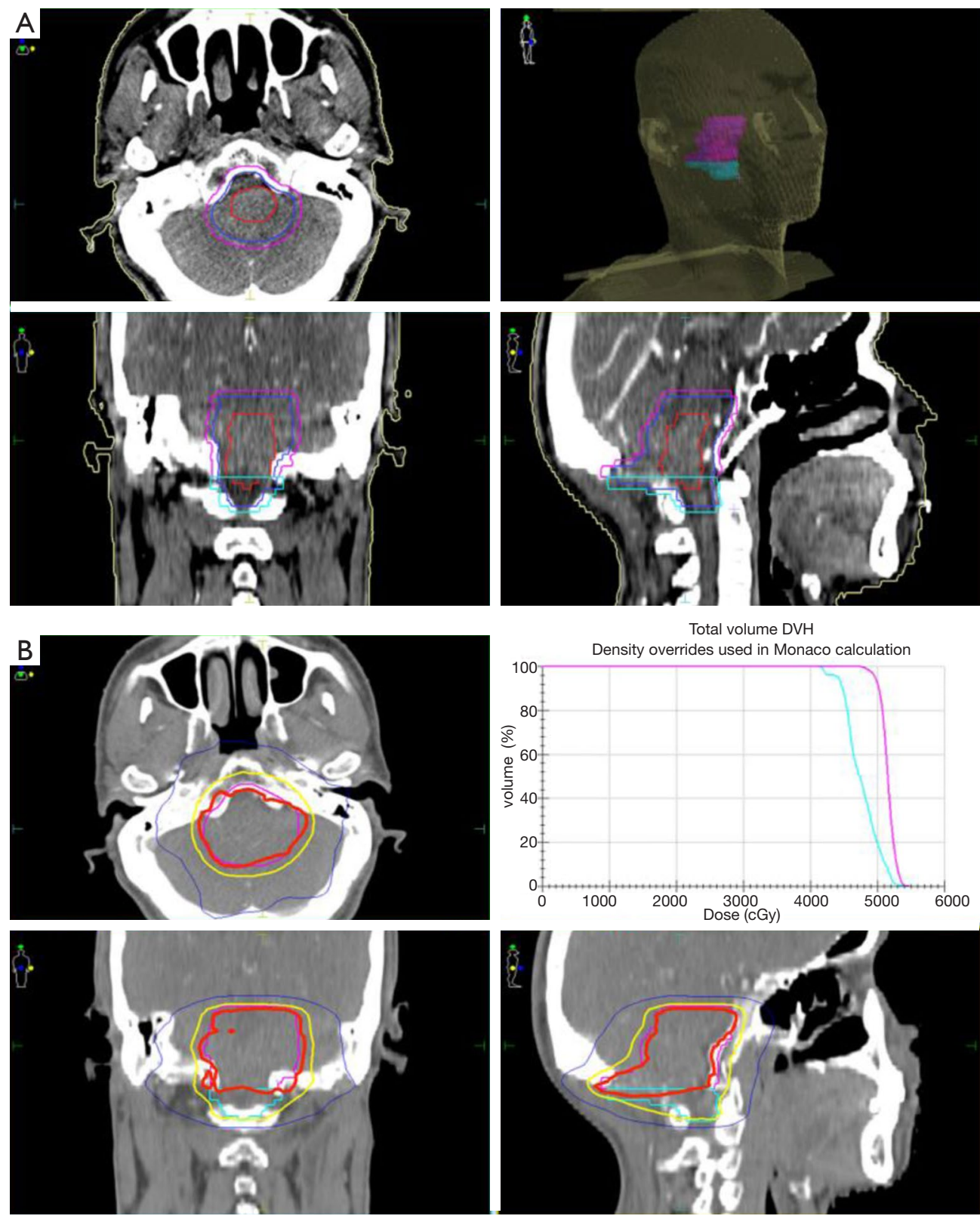

Figure 4 Radiation fields and doses for the tumor. (A) Clinical pictures of the radiation fields with red line of gross tumor volume (GTV), blue line of clinical target volume (CTV), purple and light blue lines of planning target volume (PTV). (B) Dose Volume Histogram (DVH) of the radiation doses for PTV. The PTV was composed of the light blue lines (the medullary neck mass) and the purple lines (the medullary intracranial mass).

fluid rhinorrhea (15) and dissemination of cerebrospinal fluid (16). In the only one reported GGs located in the medulla oblongata, the 7 -year-old boy was admitted to hospital with complaints of ataxia, seizure and sleep apnea (6). While according to PF location of the tumor in our case, it mainly caused symptoms of the posterior cranial nerve, including vomiting, dysphagia, choking, hiccup, hoarseness and ipsilateral limb weakness.

In imaging manifestations, a plain CT for GGs commonly exhibited features of solid and cystic components with low density, while focal calcifications of tumor capsules were also observed (17). In MRI, GGs showed equal intensity or low 
signal on T1 weighted image (T1WI) and heterogeneous high signal on $\mathrm{T} 2$ weighted image (T2WI) when compared to normal parenchyma (18). More importantly, cystic solid masses with moderate to significant enhancement mainly in dorsal and posterior margins were more common in GGs located in the medulla oblongata (6). While in astrocytomas, the imaging usually showed cystic changes with nodules and enhanced cyst wall and nodules with few calcifications in enhanced scans (19). Johnson et.al also reported that curved band lesion with low or mixed density and common calcification had few cysts and enhancement, which was usually observed in oligodendroglioma (20). With a similar presentation on T1WI and T2WI, the MRI manifestation of our case showed no significant enhancement in the enhanced images in the early period while obvious ringenhanced signal in the later recurrence period, which differed from the previously reported case of GG.

As was generally recognized, surgical removal was the preferred treatment for GGs, aiming at achieving a safe complete tumor resection (21), while radical resection was not recommended in patients with GGs in significant function sites, which would result in several function losses or even death (6). Moreover, radiotherapy was considered the best option for GGs not amenable of surgical resection or partially removed, which should be performed as soon as possible $(21,22)$. Accumulating studies have shown that epidermal growth factor receptor (EGFR), histone deacetylase 6 (HDAC6), and survivin could induce the radiosensitivity of GGs (23-25), while transketolase 1 (TKTL1) promoted radiotherapy resistance (26). In the previously reported GG in the medulla oblongata, the tumor was partially resected due to severe bradycardia during the operation. Although both radiotherapy and chemotherapy were carried out after the operation, the tumor had no significant changes (6). In our case, the surgical site was located in the core functional area accompanied by ample nourishing blood vessels, which laid great difficulty on complete surgical resection. We supposed that radical surgical resection may bring catastrophic consequences to the patient. In addition, although the effect of chemotherapy in the initial treatment of GGs is still debated, Rosselló et al. has proven that chemotherapy might be effective in recurrent and deteriorating GGs (27). Therefore, in order to ensure safety as much as possible, a partial resection combined with postoperative radiotherapy and tentative chemotherapy was selected.

The gross aspect of GGs consists mostly of firm nodular tissue of white-greyish color (28). Due to the presence of both the glial and neuronal components, the histopathologic features of GGs are moderate, inhomogeneous cellularity with marked cytonuclear pleomorphism (29). Histologically, the GFAP stain highlighted the glial components, whereas positive staining for synaptophysin, $\mathrm{CgA}$ and NeuN labeled the neuronal/ganglion cell components in the GGs (30). In addition, the immunohistochemistry of BRAF V600E, a common molecular feature of GGs, could be helpful $(31,32)$. The previously reported case had no detailed description of histological performance. Our case additionally showed positive reaction of Nestin and Olig2 and negative symbol of BRAF mutation from the pathological and immunohistochemical performance, which were the specialities of our case.

Regarding the prognosis of GG, most patients with GG have a good prognosis after complete resection (33). In the previously reported case, the patient died of respiratory arrest five months after the operation (6). Postoperative genetic testing is so comprehensive that BRAF V600E mutations and Methyl Guanine Methyl Transferase (MGMT) promoter methylation have been verified to increase the probability of tumor recurrence and have a shorter progression-free survival $(34,35)$. In our case, early surgical removal, adjuvant treatment and negative BRAF mutation brought the patient a good trend of improvement.

However, there are still some limitations with our case report. Firstly, due to the quantity of nourishing blood vessels and location of the lesion, the GG could not be completely removed, laying the incentive for the later tumor progression, while this was consistent with the individualized treatment principle of tumor patients. Secondly, effective and comprehensive intraoperative monitoring has not been applied. Thirdly, with an indefinite median survival, although the patient had a left upper limb muscle strength Grade V- with a KPS score of 90, the follow-up was insufficient, which could still provide reference for the diagnosis and treatment of GGs.

\section{Conclusions}

We have presented the rare case of a middle-aged man with a GG arising from the medulla oblongata, which has a follow-up for 33 months. Review of the literature suggests that based on signs, symptoms, and imaging, GGs in the medulla oblongata are difficult to diagnose, which brings the pre-surgical diagnosis to ambiguity. Therefore, the histopathologic features are vital for the diagnosis of GGs. Due to its special location, patients with GGs in the medulla 
oblongata have more risks in respiratory and circulatory system. The patients given early surgical removal of such tumor and adjuvant radiotherapy would have a favorable prognosis. The tentative chemotherapy might be effective when it shows anaplastic features, which needs further experiments for verification.

\section{Acknowledgments}

Funding: Young Talent Development Plan of Changzhou Health Commission (2020-233); Changzhou Health and Family Planning Commission Youth Talent Science and Technology Project (WZ201811).

\section{Footnote}

Reporting Checklist: The authors have completed the CARE reporting checklist. Available at https://dx.doi. org/10.21037/tcr-21-124

Conflicts of Interest: All authors have completed the ICMJE uniform disclosure form (available at https://dx.doi. org/10.21037/tcr-21-124). The authors have no conflicts of interest to declare.

Ethical Statement: The authors are accountable for all aspects of the work in ensuring that questions related to the accuracy or integrity of any part of the work are appropriately investigated and resolved. All procedures performed in studies involving human participants were in accordance with the ethical standards of the institutional and/or national research committee(s) and with the Helsinki Declaration (as revised in 2013). Written informed consent was obtained from the patient.

Open Access Statement: This is an Open Access article distributed in accordance with the Creative Commons Attribution-NonCommercial-NoDerivs 4.0 International License (CC BY-NC-ND 4.0), which permits the noncommercial replication and distribution of the article with the strict proviso that no changes or edits are made and the original work is properly cited (including links to both the formal publication through the relevant DOI and the license). See: https://creativecommons.org/licenses/by-nc-nd/4.0/.

\section{References}

1. Horbinski C, Kofler J, Yeaney G, et al. Isocitrate dehydrogenase 1 analysis differentiates gangliogliomas from infiltrative gliomas. Brain Pathol 2011;21:564-74.

2. Podlesek D, Geiger K, Hendry DJ, et al. Rosette-forming glioneuronal tumor of the fourth ventricle in an elderly patient. J Neurooncol 2011;103:727-731.

3. Agrawal M, Borkar SA, Khanna G, et al. Pigmented Ganglioglioma of the Cerebellum: Case Report and Review. World Neurosurg 2018;116:18-24.

4. Harrison W, Elsamadicy AA, McMahon JT, et al. Glioneuronal Tumor With Features of Ganglioglioma and Neurocytoma Arising in the Fourth Ventricle: A Report of 2 Unusual Cases and a Review of Infratentorial Gangliogliomas. J Neuropathol Exp Neurol 2019;78:780-7.

5. Das KK, Bettaswamy GP, Mehrotra A, et al. Dorsally exophytic glioblastoma arising from the medulla oblongata in an adult presenting as 4(th) ventricular mass. Asian J Neurosurg 2017;12:224-7.

6. Akiyama H, Nakamizo S, Kawamura A, et al. Ganglioglioma of the medulla oblongata: case report and review of the literature. No Shinkei Geka 2006;34:1255-60.

7. Jarius S, Kleiter I, Ruprecht K, et al. MOG-IgG in NMO and related disorders: a multicenter study of 50 patients. Part 3: Brainstem involvement - frequency, presentation and outcome. J Neuroinflammation 2016;13:281.

8. Degens $\mathrm{H}$. The role of systemic inflammation in agerelated muscle weakness and wasting. Scand J Med Sci Sports 2010;20:28-38.

9. Donson AM, Kleinschmidt-DeMasters BK, Aisner DL, et al. Pediatric brainstem gangliogliomas show BRAF(V600E) mutation in a high percentage of cases. Brain Pathol 2014;24:173-83.

10. Oushy S, Perry A, Graffeo CS, et al. Pediatric ganglioglioma of the brainstem and cervicomedullary junction: a retrospective cohort study. J Neurosurg Pediatr 2019:1-7. doi: 10.3171/2019.7.PEDS1961.

11. Tandon V, Bansal S, Chandra PS, et al. Ganglioglioma: Single-institutional experience of 24 cases with review of literature. Asian J Neurosurg 2016;11:407-411.

12. Prasad GL, Kumar R, Kurwale N, et al. Intraventricular Gangliogliomas: A Review. World Neurosurg 2016;87:39-44.

13. Vogt VL, Witt JA, Delev D, et al. Cognitive features and surgical outcome of patients with long-term epilepsyassociated tumors (LEATs) within the temporal lobe. Epilepsy Behav 2018;88:25-32.

14. Kaymak-Cihan M, Erdis E, Bozkurt S, et al. Pediatric 
primary anaplastic ganglioglioma with malignant neuronal component. Turk J Pediatr 2018;60:102-6.

15. Kandregula S, Beniwal M, Srinivas D, et al. Ganglioglioma with Cerebrospinal Fluid Rhinorrhea: A Rare Presentation. World Neurosurg 2019;127:11-4.

16. de Abreu PP, Muniz BC, Ventura N, et al. Intraventricular ganglioglioma with dissemination of cerebrospinal fluid. Radiol Bras 2018;51:272-3.

17. Dorne HL, O'Gorman AM, Melanson D. Computed tomography of intracranial gangliogliomas. AJNR Am J Neuroradiol 1986;7:281-5.

18. Cohen AR. The great neurosurgical masquerader: 3 cases of desmoplastic infantile ganglioglioma. J Neurosurg Pediatr 2019:1-9. [Epub ahead of print]. doi: 10.3171/2019.5.PEDS19151.

19. She DJ, Lu YP, Xiong J, et al. Comparison of conventional, diffusion, and perfusion MRI between infratentorial ganglioglioma and pilocytic astrocytoma. Acta Radiol 2019;60:1687-94.

20. Johnson DR, Diehn FE, Giannini C, et al. Genetically Defined Oligodendroglioma Is Characterized by Indistinct Tumor Borders at MRI. AJNR Am J Neuroradiol 2017;38:678-84.

21. Walker DA, Liu J, Kieran M, et al. A multi-disciplinary consensus statement concerning surgical approaches to low-grade, high-grade astrocytomas and diffuse intrinsic pontine gliomas in childhood (CPN Paris 2011) using the Delphi method. Neuro Oncol 2013;15:462-8.

22. Packer RJ. Radiation therapy for pediatric low-grade gliomas: survival and sequelae. Curr Neurol Neurosci Rep 2010;10:10-3.

23. Alexandru O, Purcaru SO, Tataranu LG, et al. The Influence of EGFR Inactivation on the Radiation Response in High Grade Glioma. Int J Mol Sci 2018;19:229.

24. Yang W, Liu Y, Gao R, et al. HDAC6 inhibition induces glioma stem cells differentiation and enhances cellular radiation sensitivity through the $\mathrm{SHH} / \mathrm{Gli1}$ signaling pathway. Cancer Lett 2018;415:164-76.

25. Li J, Han Y, Zhou D, et al. Downregulation of Survivin Gene Expression Affects Ionizing Radiation Resistance

Cite this article as: Han W, Guan W, Shi J. Anaplastic ganglioglioma originating from the medulla oblongata: case report. Transl Cancer Res 2021;10(6):3059-3066. doi: 10.21037/ tcr-21-124 of Human T98 Glioma Cells. Cell Mol Neurobiol 2018;38:861-8.

26. Heller S, Maurer GD, Wanka C, et al. Gene Suppression of Transketolase-Like Protein 1 (TKTL1) Sensitizes Glioma Cells to Hypoxia and Ionizing Radiation. Int J Mol Sci 2018;19:2168.

27. Rossello A, Plans G, Vidal-Sarro N, et al. Ganglioglioma Progression to Combined Anaplastic Ganglioglioma and Anaplastic Pleomorphic Xanthoastrocytoma. Case Report and Literature Review. World Neurosurg 2017;108:996. e917-996.e925.

28. Antonia-Carmen L, Tiberiu Augustin G, Diana P, et al. Grading Gangliogliomas: a Short Case Series With Clinico-Imagistic and Immunohistopathological Correlations. Maedica 2018;13:241-9.

29. Hirose T, Scheithauer BW, Lopes MB, et al. Ganglioglioma: an ultrastructural and immunohistochemical study. Cancer 1997;79:989-1003.

30. Ho CY, Bornhorst M, Almira-Suarez MI, et al. Clinicopathologic Features of Diencephalic Neuronal and Glioneuronal Tumors. J Neuropathol Exp Neurol 2020;79:67-73.

31. Merlin MS, Gilson P, Rouyer M, et al. Rapid fullyautomated assay for routine molecular diagnosis of BRAF mutations for personalized therapy of low grade gliomas. Pediatr Hematol Oncol 2020;37:29-40.

32. Sugiura $Y$, Nagaishi M. Clinical relevance of BRAF status in glial and glioneuronal tumors: A systematic review. J Clin Neurosci 2019;66:196-201.

33. Garnier L, Ducray F, Verlut C, et al. Prolonged Response Induced by Single Agent Vemurafenib in a BRAF V600E Spinal Ganglioglioma: A Case Report and Review of the Literature. Front Oncol 2019;9:177.

34. Chen X, Pan C, Zhang P, et al. BRAF V600E mutation is a significant prognosticator of the tumour regrowth rate in brainstem gangliogliomas. J Clin Neurosci 2017;46:50-7.

35. Wang L, Li Z, Liu C, et al. Comparative assessment of three methods to analyze MGMT methylation status in a series of 350 gliomas and gangliogliomas. Pathol Res Pract 2017;213:1489-93. 